\title{
МОДЕЛИРОВАНИЕ ДИНАМИКИ ПОКАЗАТЕЛЕЙ РЫНКА МОБИЛЬНОЙ СВЯЗИ
}

\author{
Варшавский Л.Е.
}

В статье рассматриваются методы моделирования динамики показателей олигополистического рынка мобильной связи (на примере рынка США). С использованием методов теории динамических игр рассматриваются средне- и долгосрочные последствия возможных структурных изменений на рынке в результате возможного объединения компаний-провайдеров. Путем компьютерного моделирования исследовань условия, при которых может быть повышена рыночная доля объединенной компании.

DOI: $10.20537 /$ mce2019econ11

1. Введение. Характерной чертой развития рынка мобильной беспроводной связи в последнее десятилетие, как во всем мире, так и в США, является преимущественный рост его масштабов за счет быстрого роста трафика данных после перехода в начале 2000-х гг. на технологию 3G, а затем, - на технологию 4G. Так, в 2012 г. на долю технологии 4G, осваивающейся с 2010 г., приходилось 14\% всего мобильного трафика данных в мире, хотя ее доля в общем числе подключений (connections) составляла всего 1\% [1]. В 2016 г. доля этой технологии в общемировом мобильном трафике данных составляла уже $69 \%$ при приходящейся на нее доле подключений $26 \%$ [2].

О высоких темпах роста мобильного трафика в США свидетельствуют следующие данные. Если в 2011 г. доля мобильного трафика в общих доходах компаний отрасли составляла немногим более одной трети, что было существенно ниже доли голосовой связи (63\%), то уже в 2014 г. она превысила 50\%. В 2017 г. объем мобильного трафика данных в США составил 15687 млрд. МВ (т.е. $15.687 * 10^{18}$ байт), что в 40 раз выше, чем в 2010 г. [3-5]. При этом интересно отметить, что в США в последние годы соотношение между общим числом подключений $Q_{t}$ (connections, subscribers) и десятичным логарифмом трафика 
$\log _{10}\left(\operatorname{Traffic}_{t}\right)$ оставалось относительно стабильным, и в среднем в 20132017 гг. составляло $Q_{t} / \log _{10}\left(\right.$ Traffic $\left._{t}\right)=99.5$ млн. соединений на МВ.

В ближайшей перспективе доля трафика данных в общих доходах компаний отрасли может возрасти до $75 \%$. Дальнейший рост этой доли возможен при переходе к технологии $5 \mathrm{G}$, хотя такой переход потребует решения ряда серьезных проблем.

Рост активности в отрасли мобильной связи США сопровождается повышением рыночной доли крупных компаний-провайдеров. Так, на долю 4-х компаний (Verizon, AT\&T, T-Mobile и Sprint) приходится около $100 \%$ всех подключений и доходов от мобильного сервиса в США, причем доля этих компаний в подключениях в сложившейся квадрополии составляла в 2017 г. соответственно $35.5 \%, 34.3 \%, 17.3 \%$ и $12.8 \%$.

В настоящее время в Федеральной комиссии США по связи (Federal Communications Commission, сокр. FCC) обсуждается проблема слияния T-Mobile и Sprint. Противники объединения опасаются усиления концентрации на рынке всего 3-х компаний (возникнет триополия). В свою очередь, сторонники слияния отмечают в качестве положительного факта то, что объединенная компания будет более сильным конкурентам для AT\&T и Verizon. Кроме того, они считают, что в результате слияния будет происходить более быстрое развертывание системы $5 \mathrm{G}$ (см., напр., [6]).

Проблема формирования эффективной структуры рынка мобильных сервисов стоит и в нашей стране, где также высока доля концентрации услуг в небольшом числе компаний-провайдеров. В связи с этим, представляет практический интерес разработка методических подходов к исследованию перспективной структуры этого рынка.

В настоящей статье на основе динамической игровой модели проводится исследование перспективной динамики показателей рынка мобильной связи США как при наличии 4 компаний $(\mathrm{N}=4)$, так и при слиянии T-Mobile и Sprint, и образовании триополии $(\mathrm{N}=3)$.

2. Игровая модель. Используемая модель состоит из следующих блоков:

1. динамики соотношения между объемами капитальных вложений (Сарех в млн долл.) и числом мобильных подключений $Q_{i t}$ в і-й компании (в млн.):

$$
Q_{i t}=W_{i}(z) u_{i t}=\frac{\gamma_{i} z}{\left(z-\lambda_{i}\right)^{2}} u_{i t}+Q_{0 i t}
$$


где $\mathrm{z}$ - оператор сдвига, т.е. $z x_{t}=x_{t+1}, u_{i t}=$ Capex $_{i t}, 0<\lambda_{i}<1, Q_{0 i t}-$ объем производства при отсутствии инвестиций, $\mathrm{i}=1,2 \ldots \mathrm{N}$.

2. обратной функции спроса, связывающей среднегодовую цену в олигополии $P_{t}$ (в долл.) с суммарным числом подключений (в млн):

$$
P_{t}=a-b \sum_{i} Q_{i t}
$$

Предполагается, что олигополисты используют скользящее планирование и в каждый момент времени $\tau$ максимизируют чистую текущую стоимость (NPV):

$$
J_{\tau i}=\sum_{t=\tau}^{\tau+T_{p}} \beta^{t}\left[\left(f_{i} m_{i} p_{t}\right) Q_{i t}-u_{i t}-\frac{1}{2} \rho_{i} u_{i t}^{2}\right] \rightarrow \max _{u_{i t}},
$$

где $\beta$ - дисконт-фактор, $f_{i}$ - соотношение между ценой средними доходами на 1 соединение в і-й компании и соответствующим показателей для суммы компаний, $m_{i}$ среднее значение EBITDA margins (удельная прибыль без учета процентных платежей, налогов, амортизации; англ. earnings before interest, tax, depreciation and amortization) в і-й компании в расчете на 1 подключение, $\rho_{i}-$ коэффициенты при затратах регулирования (adjustment costs), характеризующих наличие инвестиционных ограничений. Управляющими переменными в модели (1)-(3) являются капитальные вложения $u_{t}, \mathrm{i}=1,2 \ldots \mathrm{N}$. Задача состоит в определении оптимальных по Нэшу игровых стратегий компаний-провайдеров.

Следует отметить, что идентификация составных блоков рассматриваемой модели рынка мобильной связи в США (как, впрочем, и в других странах) сопряжена с трудностями, обусловленными различием статистических данных, приводимых как официальными, так и частными организациями. В настоящей работе использовались данные годовых докладов Федеральной комиссии по связи США за 2009-2017 гг. Оценки параметров соотношений (1) и (2), полученные путем использования соответственно нелинейного и линейного метода наименьших квадратов, приведены в табл. 1 и 2 (в нижней строке табл. 2 указаны значения среднеквадратических отклонений оценок параметров). В качестве оценок $m_{i}$ приняты среднегодовые значения удельной прибыли за 20132017 гг. (табл. 3). Для получения оценок коэффициентов $\rho_{i}$ использован подход, изложенный ниже в п. 3 (см. также $[7,8]$ ).

Возможность использования эконометрических моделей при прогнозировании показателей быстро развивающегося исследуемого рынка 
обусловлена тем, что значительную часть инфраструктуры, созданной в США для технологии связи 4G, предполагается использовать и для технологии $5 \mathrm{G}$, на распространение которой в ближайшее время ориентированы компании-провайдеры [9].

Таблица 1. Значения параметров зависимости (1).

\begin{tabular}{|l|c|c|c|}
\hline Компания & $\gamma$ & $\lambda$ & Коэффициент вариачии $v$ \\
\hline Verizon & 1.198 & 0.739 & 0.085 \\
\hline AT\&T & 1.118 & 0.739 & 0.085 \\
\hline T-Mobile & 4.422 & 0.502 & 0.064 \\
\hline Sprint & 0.654 & 0.752 & 0.024 \\
\hline T-Mobile + Sprint & 1.666 & 0.708 & 0.045 \\
\hline
\end{tabular}

Таблица 2. Значения параметров зависимости (2).

\begin{tabular}{|l|l|l|}
\hline $\mathrm{a}$ & $\mathrm{b}$ & $\mathrm{R}^{2}$ \\
\hline 856.110 & 1.012 & 0.939 \\
\hline 33.314 & 0.097 & \\
\hline
\end{tabular}

Ниже на основе игровой модели (1)-(3) исследуется динамика показателей олигополистического рынка мобильной связи США на дальнюю перспективу (2030 г.), соответствующая следующим сценариям:

1. обеспечение компаниями-провайдерами наиболее выгодной по критерию NPV траектории достижения гипотетических целевых уровней подключений к 2030 г. $Q_{\text {iळ }}^{*}$ которые определяются, как $Q_{i \infty}^{*}=\frac{\gamma_{i}}{\left(1-\lambda_{i}\right)^{2}} u_{\text {iavg }}$, где $u_{\text {iavg }}-$ среднегодовые за последние 7-8 лет объемы капитальных вложений (экстраполяционный сценарий);

2. достижение желаемых гипотетических рыночных долей компаний к 2030 г., согласованных с их инвестиционными возможностями и оптимизацией NPV, при различных сценариях роста суммарного по стране мобильного трафика данных Traffic $_{t}$ (является экзогенной переменной).

Таблица 3. Принятые в модели значения $m_{i}$ (EBITDA Margins)

\begin{tabular}{|c|c|c|c|}
\hline Verizon & AT\&T & T-Mobile & Sprint \\
\hline 0.530 & 0.447 & 0.287 & 0.266 \\
\hline
\end{tabular}


3. Особенности проведения расчетов. Для проведения прогнозных расчетов на основе модели (1)-(3) при $T_{p} \rightarrow \infty$ могут быть использованы 2 подхода: 1) основанный на представлении модели в пространстве состояний и использовании обобщенных (generalized, в англоязычной литературе используются также термин coupled) матричных уравнений Риккати (см., например, $[10,11])$; 2) основанный на использовании операционного исчисления (Z-преобразования, см., например, $[12,13])$. Эти подходы взаимно дополняют друг друга.

При использовании первого подхода для расчета как разомкнутых (open-loop), так и замкнутых (feedback или Markov perfect) оптимальных по Нэшу-Курно стратегий следует предварительно представить модель (1)-(3) в эквивалентной форме в пространстве состояний:

$$
\begin{aligned}
& X_{t}=A X_{t-1}+\sum_{i=1}^{N} B_{i} u_{i t}+D \xi_{t}, \\
& J_{i}=\sum_{t=0}^{\infty} \beta^{t}\left(\frac{1}{2} X_{t}{ }^{\prime} H_{i} X_{t}-C_{0 i}{ }^{\prime} X_{t}-q_{i t}{ }^{\prime} u_{i t}-\frac{1}{2} \rho_{i} u_{i t}{ }^{2}\right) \rightarrow \max _{u_{i t}},
\end{aligned}
$$

где матрицы и векторы $A, B_{i}, D, H_{i}, C_{0 i}, q_{i t}, X_{t}, \xi_{t}, i=1,2 \ldots N$ связаны с параметрами и переменными исходной модели. Получаемые оптимальные стратегии участников олигополии $u_{i t}$ линейно связаны с вектором состояния системы (4):

$$
u_{i t}=K_{i t} X_{t-1}+\eta_{i t}
$$

в котором $K_{i t}$ и $\eta_{i t}$ - векторы, зависят от решений обобщенных уравнений Риккати [10].

При исследовании оптимальных разомкнутых (open-loop) игровых стратегий олигополистов с критерием (5), наглядным и удобным для экономического анализа является второй подход, основанный на использовании операционного исчисления и нахождении экстремума функционалов в гильбертовом пространстве. Используя этот подход, можно получить формулы для расчета оптимального управления $\mathrm{u}_{\mathrm{it}} \mathrm{u}$ объемов производства $Q_{i t}$ і-го олиголиста, максимизирующего критерий NPV с учетом затрат регулирования, а также средней цены $P_{t}$ (см. [14]):

$$
Q_{i t}=W_{i}(z) u_{i t}=\frac{\Gamma_{i}\left(z,(\beta z)^{-1}\right)}{b}\left(p_{t}-P L_{i} / m_{i}\right)
$$

где: $P L_{i}=1 / W\left(1+r_{i}\right)$; 


$$
\begin{gathered}
\Gamma_{i}\left[z,(\beta z)^{-1}\right]=\frac{b m_{i} W_{i}(z) W_{i}\left((\beta z)^{-1}\right)}{\rho_{i}+b m W_{i}(z) W_{i}\left((\beta z)^{-1}\right)}, i=1,2, \ldots N \\
P_{t}=\frac{1}{1+\sum_{i=1}^{N} \Gamma_{i}\left[z,(\beta z)^{-1}\right]}\left\{a+\sum_{i=1}^{N} \Gamma_{i}\left[z,(\beta z)^{-1}\right] P L_{i} / m\right\} .
\end{gathered}
$$

(в формулах (7)-(9) принято, что $\left.Q_{0 i t} \equiv 0, f_{i}=1, \mathrm{i}=1,2 \ldots \mathrm{N}\right)$.

Приведенные соотношения после проведения некоторых преобразований могут быть использованы при экономическом анализе стратегий участников рынка, и, в частности, при оценке ключевых параметров модели - коэффициентов $\rho_{i}$ при затратах регулирования.

Так, нетрудно показать, что норма оператора $\Gamma_{i}\left[z,(\beta z)^{-1}\right](8)$ меньше 1 , т.е. для любой суммируемой функции времени $\eta_{t}$ справедливо:

$$
\sum_{t=0}^{\infty} \beta^{t} \eta_{t} \Gamma_{i}\left[z,(\beta z)^{-1}\right] \eta_{t}=\left(\eta_{t}, \Gamma_{i}\left[z,(\beta z)^{-1}\right] \eta_{t}\right) \leq\left\|\Gamma_{i}\right\|_{\infty} *\left\|\eta_{t}\right\|^{2} \leq\left\|\eta_{t}\right\|^{2}
$$

Тогда из (7) и (10) следует:

$$
\sum_{t=0}^{\infty} \beta^{t} Q_{i t}{ }^{2}=\left\|Q_{i t}\right\|_{2}^{2} \leq \sum_{t=0}^{\infty} \beta^{t}\left(\frac{p_{t}-P L_{i} / m_{i}}{b}\right)^{2}=\left\|\frac{p_{t}-P L_{i} / m_{i}}{b}\right\|_{2}^{2},
$$

Таким образом, величина соотношения

$$
\chi_{i}=\frac{\sum_{t=0}^{T} \beta^{t} Q_{i t}{ }^{2}}{\sum_{t=0}^{T} \beta^{t}\left(\frac{p_{t}-P L_{i} / m_{i}}{b}\right)^{2}},
$$

которую можно оценить эмпирически, при достаточно больших значениях $T$ может быть использована для исследования согласованности поведения участников рынка с критерием оптимальности (3).

Очевидно, при $\chi_{i}>1$ поведение $i$-ой компании не согласуется с критерием NPV (3). Если же $0<\chi_{i}<1$, то можно считать, что у компании существует потенциал оптимального наращивания производства за счет 
смягчения инвестиционных ограничений, который может быть реализован путем уменьшения коэффициента $\rho_{i}$.

Для вычисления коэффициентов $\rho_{i}$ может быть использовано следующее соотношение между установившимися уровнями производства участников рынка $Q_{i \infty}$ и ценой, которое справедливо при постоянных $P L_{i}[7,8]$ :

$$
Q_{i \infty}=\frac{\Gamma_{i}(1,(1+r))}{b}\left(p_{\infty}-P L_{i} / m_{i}\right)
$$

С учетом (8) значения коэффициентов $\rho_{i}$, при которых достигаются желаемые (целевые) установившиеся уровни производства участников рынка $Q_{i \infty}$ могут быть определены по формуле:

$$
\rho_{i}=\left(\delta_{i}-1\right) b m_{i} W_{i}(1) W_{i}(1+r),
$$

где $\delta_{i}=\frac{1}{\Gamma_{i}(1,(1+r))}=\frac{\left(p_{\infty}-P L_{i} / m_{i}\right)}{b Q_{i \infty}}-$ характеристика инвестиционных ограничений і-й фирмы (в статической модели Курно, в которой отсутствуют такие ограничения, $\left.\delta_{i}=1\right) i=1,2, \ldots N$. После нахождения коэффициентов $\rho_{i}$ может быть рассчитана прогнозная динамика объемов производства олигополистов $Q_{i t}$, цены и других рыночных показателей.

С другой стороны, на основе соотношений (12)-(13) можно приближенно оценить фактические значения коэффициентов $\rho_{i}$ за ретроспективный период. Для этого в соотношении (13) следует вместо $\delta_{i}=\frac{1}{\Gamma_{i}(1,(1+r))}$ использовать $1 / \sqrt{\chi_{i}}$. Во многих случаях, близкие к таким оценкам значения $\chi_{i}$ и $\rho_{i}$ можно получить путем замены сумм в числителе и знаменателе (12) на среднегодовые значения этих показателей за представительный период: $Q_{i \text { avg }}$ и $p_{\text {avg }}$.

Следует, однако, отметить, что использование получаемых в последних случаях значений коэффициентов $\rho_{i}$ предпочтительно при проведении прогнозов показателей устоявшихся рынков традиционной продукции. При прогнозировании показателей быстро развивающихся рынков получаемые таким образом оценки могут приводить к заниженным прогнозным уровням производства $Q_{i t}$. Так, на рис. 1 представлены 
Вариавский Л.Е. Моделирование динамики показателей рынка мобильной.., стр. 116-131 Varshavsky L.E. Modeling dynamics of indicators of mobile wireless..., pp. 116-131

траектории показателя подключений компаний-провайдеров США при использовании приближенных оценок величин $\chi_{i}$ и $\rho_{i}$, полученных по данным за период 2009-2017 гг. (табл. 4). Изображенные на рис.1 уровни подключений не соответствуют фактической возрастающей динамике этого показателя для первых трех компаний за последние 3 года.

Таблица 4. Значения параметров $\chi_{i}$ и $\rho_{i}$

\begin{tabular}{|l|c|c|c|c|}
\hline Параметр & Verizon Wireless & AT\&T & T-Mobile & Sprint \\
\hline$\chi_{i}$ & 0.077 & 0.113 & 0.038 & 0.875 \\
\hline$\rho_{i}$ & $3.586 \cdot 10^{-4}$ & $2.002 \cdot 10^{-4}$ & $3.501 \cdot 10^{-4}$ & $1.737 \cdot 10^{-6}$ \\
\hline
\end{tabular}

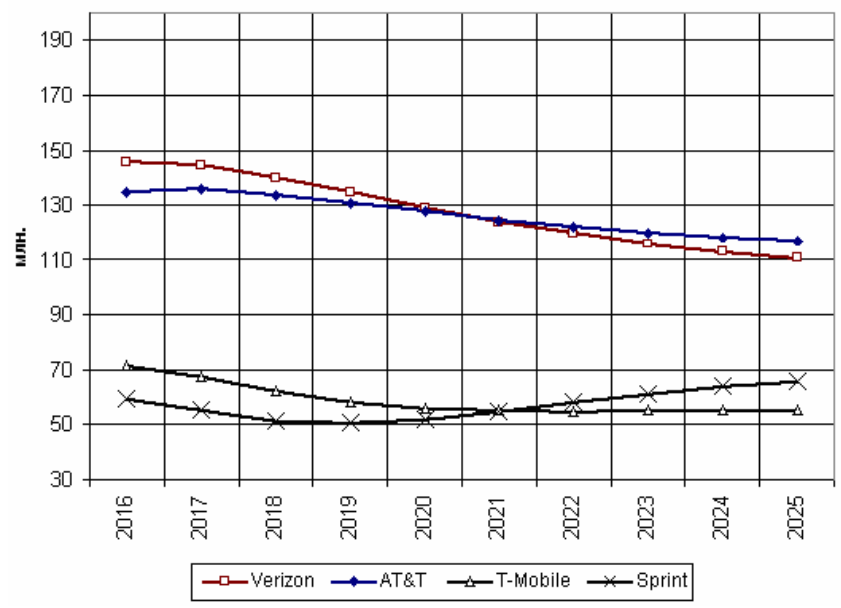

Рис.1. Динамика числа подключений 4-х компаний-провайдеров при значениях коэффициентов $\rho_{i}$ из табл. 4.

В связи с этим, в настоящей статье при определении разомкнутых (open-loop) оптимальных по Нэшу стратегий участников рынка, а также средней цены мобильных сервисов, использовались оценки коэффициентов $\rho_{i}$, получаемые на основе (13) с учетом гипотетических желаемых уровней подключений $Q_{i}^{*}$ и $Q_{i 2030}$. Расчет оптимальных стратегий проводился на основе обобщенных уравнений Риккати для линейных динамических игр с квадратичным критерием [10]. 


\section{4. Результаты расчетов.}

a) 4 участника рынка. В экстраполяционном сценарии оптимальная в соответствии с критерием NPV динамика числа подключений 4-х компаний-провайдеров, согласованная с заданными на 2030 г. целями $Q_{i}^{*}$ (соответственно 174 млн, 166 млн, 70 млн и 38 млн подключений), представлена на рис. 2. При этих гипотетических целях компаний значения коэффициента и $\Gamma_{i}(1,(1+r))$ будут меньше 1 для всех компаний, кроме компании Sprint, имеющей наихудшие экономические показатели прибыльности. Поэтому для компании Sprint здесь и далее использовался критерий (3) при $m_{i}=1$, т.е. одна из форм критерия максимизации дохода (Revenue).

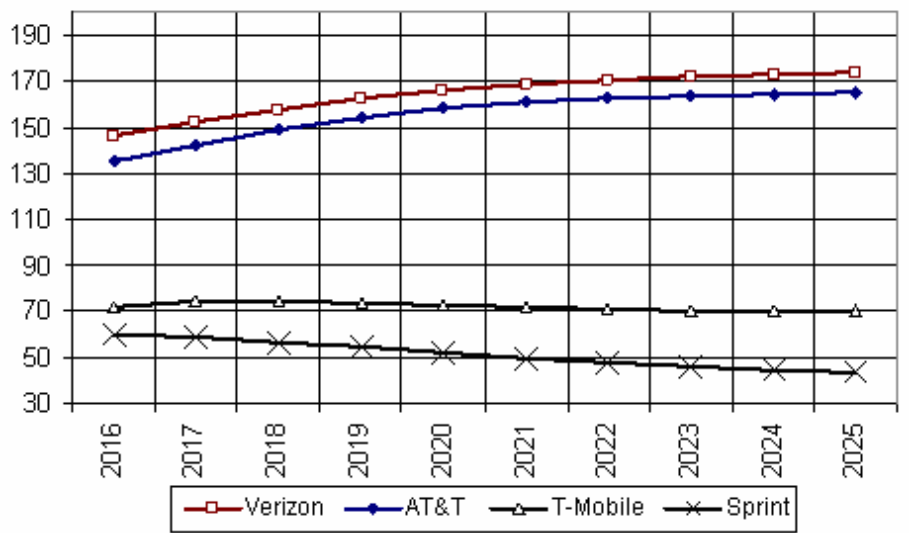

Рис.2. Динамика числа подключений 4-х компаний-провайдеров, согласованная с гипотетическими целями на 2030 г. (экстраполяционный сценарий).

Рост числа подключений будет иметь место лишь в компанияхлидерах (Verizon, AT\&T). Ввиду невысоких объемов капитальных вложений в компании Sprint, число подключений в этом провайдере должно уменьшиться с 59 млн в 2017 г. до 43 млн в 2025 г. В компании ТMobile в соответствии с принятой гипотезой, уровень подключений, несмотря на рост капитальных вложений в последние годы, должен немного снизиться (до 70 млн). 
Оптимальная динамика числа подключений компаний $Q_{i t}$, а также удельных доходов на 1 подключение ARPU (average return per unit) при различных сценариях роста мобильного трафика данных (с темпом прироста $5 \%, 10 \%, 15 \%$ ), соответствующая сохранению рыночных долей компаний по показателю подключений к 2030 г. на уровне 2017 г. (соответственно $0.355 ; 0.343 ; 0.173 ; 0.128)$, представлена на рис. $3-5$. При этом использовано следующее устойчиво сохраняющееся в последние 5 лет регрессионное соотношение между суммарным числом подключений в 4 компаниях (в млн.) и мобильным трафиком (в млрд. МВ): $Q_{\Sigma t}=99.5 * \log _{10}\left(\operatorname{Traffic}_{\Sigma t}\right)$.

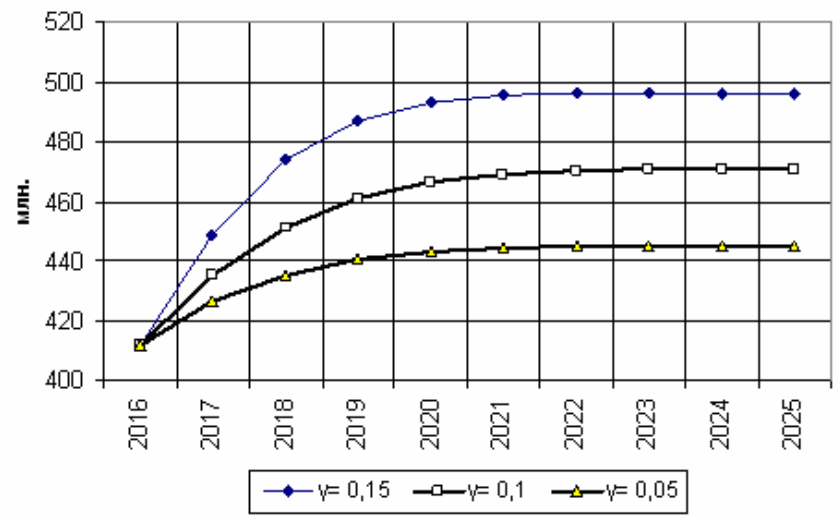

Рис.3. Динамика суммарного числа подключений 4-х компаний-провайдеров при различных сценариях (вариантах) роста трафика данных.

Как и следовало ожидать, в этих сценариях компании Verizon и AT\&T сохраняют лидирующие позиции. Однако в сценарии $15 \%$-го роста мобильного трафика 2 компании-аутсайдеры приближаются к каждому из лидеров по суммарному числу подключений (рис. 4). 
Анализ и моделирование экономических и сочиальных прочессов - МКО - 2019 Analysis and modeling of economic and social processes - MCE - 2019

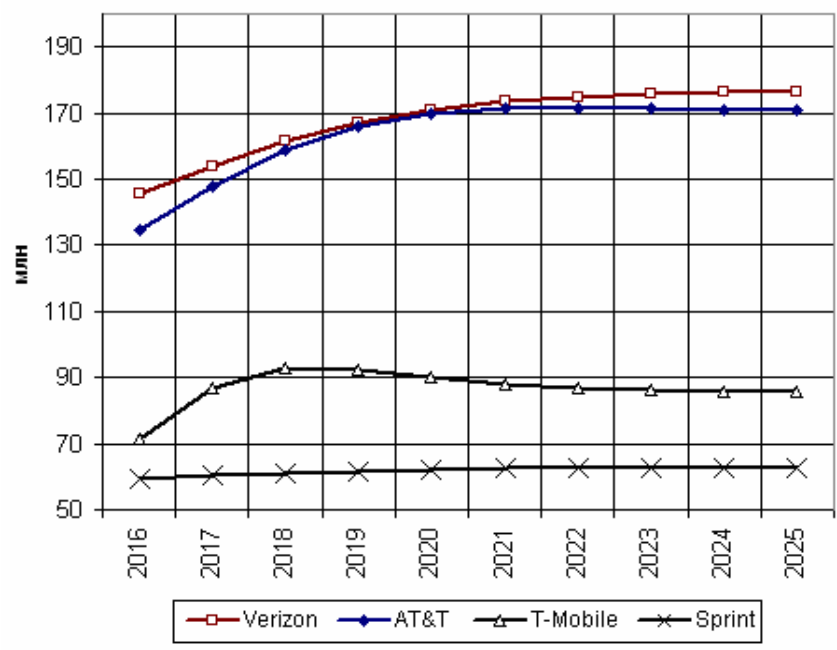

Рис.4. Динамика числа подключений 4-х компаний-провайдеров при сценарии 15\%-го роста трафика данных.

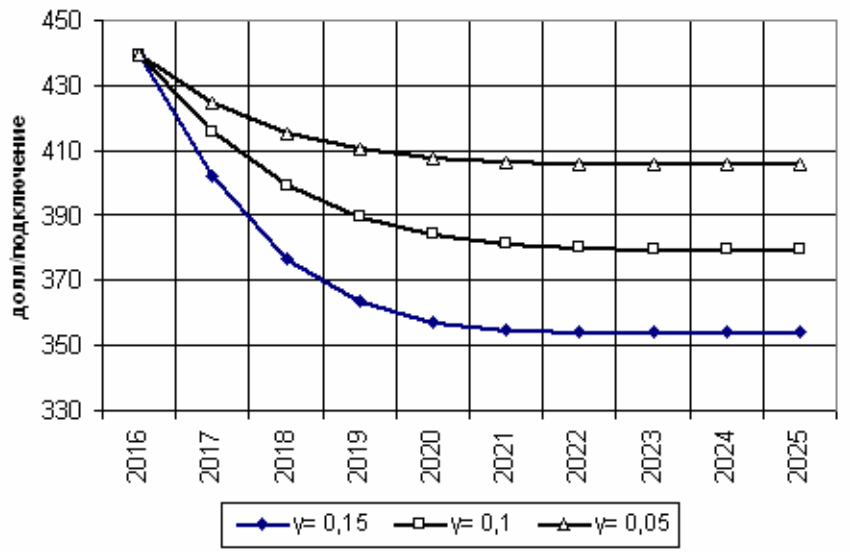

Pис.5. Динамика удельных доходов на 1 подключение ARPU при различных сценариях (вариантах) роста трафика данных. 
б) Влияние слияния компаний на показатели рынка. Реализация исследуемых сценариев в триополии (Verizon, AT\&T, TMobile+Sprint) может привести к более равномерному распределению числа подключений между компаниями, чем в случае квадрополии. Это связано с возможностью проявления синергического эффекта при слиянии T-Mobile, наращивающей в последние годы объемы капиталовложений, с компанией Sprint (pис. 6).

Следует, однако, отметить, что при рассматриваемом относительно умеренном темпе прироста мобильного трафика в $15 \%$ и соответствующих ему целевых уровнях подключений компаний $Q_{i 2030}$ (соответственно 176 млн, 170 млн и 149 млн) оптимальная по критерию NPV (3) стратегия объединенной компании может быть достигнута лишь при достаточно высоком значении удельной прибыли (порядка $40 \%$, т.е. при $\left.m_{3}=0.4\right)$. Это значение маржи $m_{3}$ для T-Mobile+Sprint принято в дальнейших расчетах.

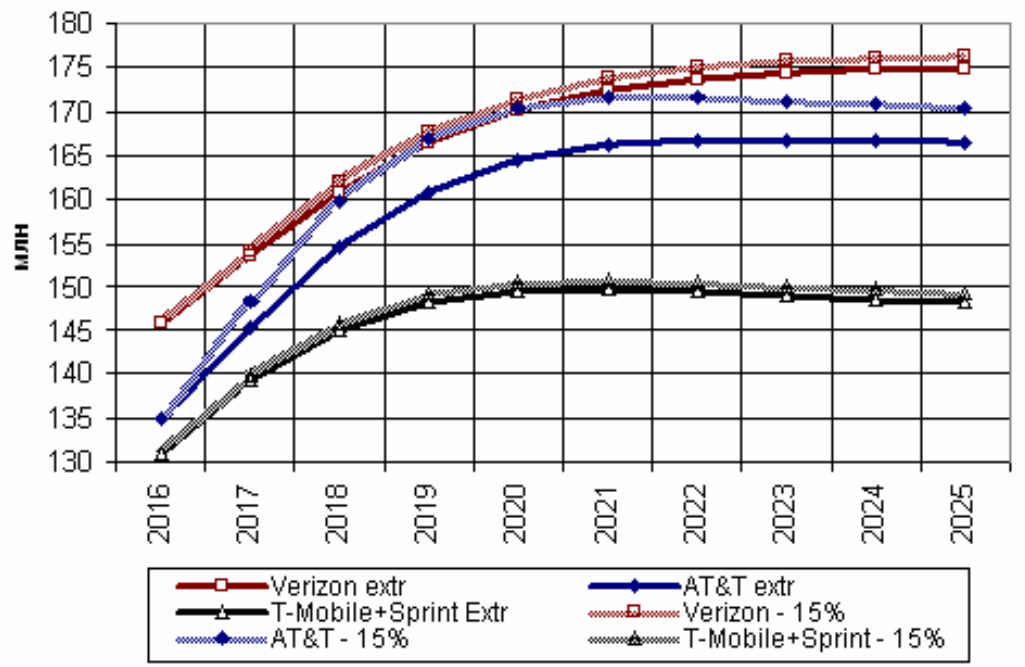

Рис.6. Динамика числа подключений в триополии при экстраполяционном сценарии (extr) и при сценарии 15\%-го роста трафика данных (15\%). 


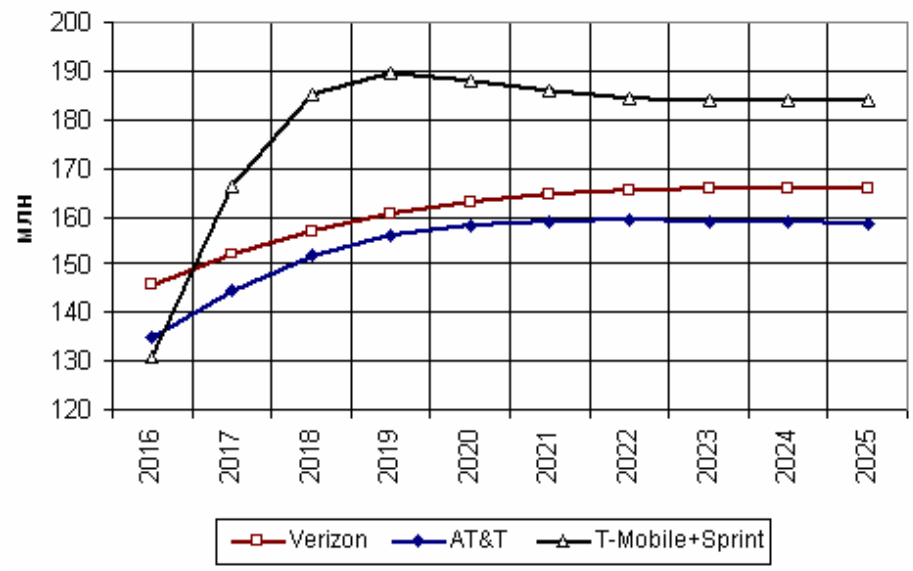

Рис.7. Динамика числа подключений в компаниях триополии при отклонении целей компаний от первоначально принятых (вариант 1 , сценарий $15 \%$-го роста трафика данных).

Интересно также отметить близость динамики и числа подключений в сценарии 15\%-го роста мобильного трафика и в экстраполяционном сценарии как для компании Verizon, так и для объединенной компании Mobile+Sprint (рис. 5).

Вместе с тем, расчеты показывают, что в результате слияния новая объединенная компания может захватить значительную долю рынка, отклонившись от целей, ожидаемых другими компаниями. Так, при ориентации этой компании на достижение $35 \%$-й доли на рынке в 2030 г. (вместо предполагаемой другими участниками рынка $30.1 \%$, при целевых установках конкурентов на прежних уровнях: $35.5 \%$ и $34.3 \%$ ) ее рыночная доля уже к 2025 г. составит более $36 \%$ (рис. 7, вариант 1).

При другом гипотетическом варианте событий, когда Verizon попытается, достигнуть $40 \%$ доли рынка, а AТ\&Т и Т-Mobile+Sprint - 35\%й доли рынка каждый (полагая, что конкуренты будут ориентироваться на свои старые цели), новая объединенная компания, увеличив свою рыночную долю до 33.2\%, несколько потеснит АТ\&Т. Рыночная доля последней составит $29.4 \%$ (рис. 8 , вариант 2). Таким образом, объединение компаний-аутсайдеров может, при условии повышения своей эффективности 
(в частности, удельной прибыли $m_{3}$ ), представлять угрозу для нынешних бесспорных лидеров американского рынка мобильной связи.

Однако существует риск того, что дальнейшее широкомасштабное распространение мобильной связи в США, особенно освоение технологии 5G, может столкнуться с существенными трудностями, Это связано с недостаточной маркетинговой проработкой эффективности возможных направлений технологии мобильной связи $5 \mathrm{G}$, а также с неисследованностью последствий влияния значительного увеличения плотности и интенсивности сверхвысокочастотного электромагнитного излучения на здоровье населения и на инфраструктуру.

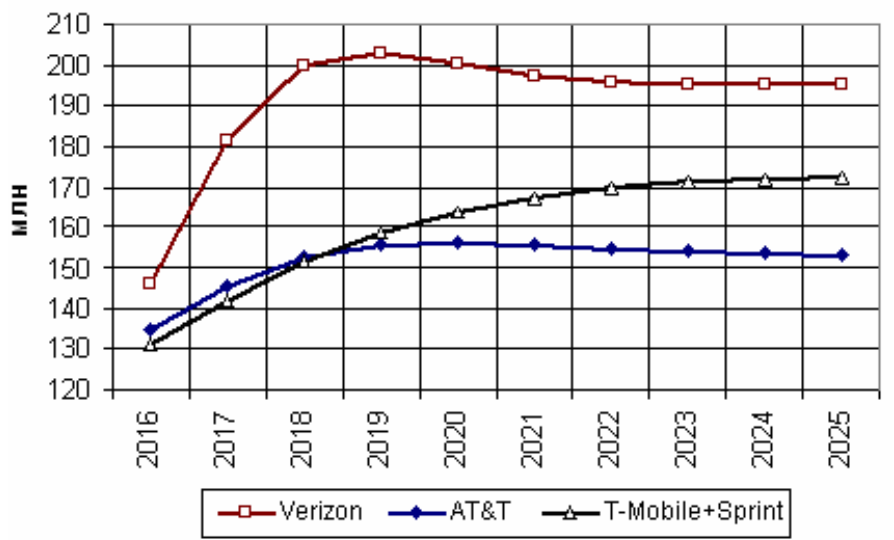

Рис.8. Динамика числа подключений в компаниях триополии при отклонении целей компаний от первоначально принятых (вариант 2 , сценарий $15 \%$-го роста трафика данных).

Выводы. Объединение компаний T-Mobile и Sprint может существенно повлиять на структуру рынка мобильной связи в США в сторону повышения рыночной доли объединенной компании.

Использование линейных динамических игр с квадратичным критерием позволяет провести содержательный анализ стратегий компаний-провайдеров и разработать сценарии и прогнозы динамики показателей олигополистических рынков мобильной связи как в зарубежных странах, так и в России. 
Существует риск того, что дальнейшее широкомасштабное распространение мобильной связи в США, особенно освоение технологии $5 \mathrm{G}$, может столкнуться с существенными трудностями, обусловленными недостаточной маркетинговой проработкой направлений использования 5G и недоучетом медицинских факторов.

\section{СПИСОК ЛИТЕРАТУРЫ}

1. Maternia M. Deployment and Transition of Telecommunication Systems / In The Telecommunications Handbook: Engineering Guidelines for Fixed, Mobile and Satellite Systems, Edited by J. T. J. Penttinen. John Wiley \& Sons, 2015.

2. Cisco Visual Networking Index.

URL: https://www.cisco.com/c/en/us/solutions/collateral/service-provider/visualnetworking-index-vni/mobile-white-paper-c11-520862.html.

3. Annual Report and Analysis of Competitive Market Conditions With Respect to Mobile Wireless, Including Commercial Mobile Services. Sixteenth report. Federal Communications Commission FCC 13-34. Released: March 21, 2013.

4. U.S. mobile data revenue surpasses voice call revenue: analyst.

URL: https://www.reuters.com/article/us-usa-mobilephone-data/u-s-mobiledata-revenue-surpasses-voice-call-revenue-analyst-idUSBREA2C24J20140313.

5. The wireless industry data.

URL: https://www.ctia.org/the-wireless-industry/infographics-library.

6. T-Mobile/Sprint Merger Offers Public Interest Benefits: Likely Presents a Fast Track to 5G // Perspectives from FSF Scholars. July 12, 2018. Vol. 13, No. 27.

URL: http://www.freestatefoundation.org/images/T-MobileSprint_Merger_Offers_Public_Interest_Benefits_071218.pdf .

7. Варшавский Л.Е. Экономико-математические методы анализа динамики рыночных процессов и формирования конкурентной среды в микроэлектронной промышленности // Национальные интересы: приоритеты и безопасность. 2016. №336. с. 26-44.

8. Варшавский Л.Е. Моделирование динамики ключевых показателей рынков компонентов высокопроизводительных вычислительных систем // Труды Института системного анализа Российской академии наук. 2017. №1 (66). с. 12-27.

9. 5G: The new network arrives TMT Predictions 2019.

URL: https://www2.deloitte.com/insights/us/en/industry/technology/technologymedia-and-telecom-predictions $/ 5 \mathrm{~g}$-wireless-technology-market.html.

10. Basar T., Olsder G.J. Dynamic Noncooperative Game Theory. London/New York: Academic Press, 1995. 
11. Dockner E.J., Jorgenson S. et. al. Differential Games in Economics and Management Science. Cambridge: Cambridge University Press, 2000.

12. Кузин Л.Т. Расчет и проектирование дискретных систем управления. М.: Машгиз, 1962.

13. Jury E.I. Theory and Applications of the Z-Transform Method. John Wiley, NY, 1964.

14. Варшавский Л.Е. Методы расчета оптимальных по Нэшу стратегий участников динамических игр на основе электронных таблиц //Анализ и моделирование экономических и социальных процессов / Математика. Компьютер. Образование: Сб. научных трудов. Выпуск 22. №3. - М.-Ижевск: Институт компьютерных исследований, 2015. с. 84-96.

\title{
MODELING DYNAMICS OF INDICATORS OF MOBILE WIRELESS COMMUNICATION MARKET (THE CASE OF THE US MOBILE WIRELESS COMMUNICATION MARKET)
}

\author{
Varshavsky L.E.
}

Methods of modeling dynamics of indicators of oligopolistic mobile wireless communication market (the case of US mobile wireless communication market) are analyzed. Prospects and consequences of structural changes on the US market over middle range and long-run periods are studied on the basis of methods of dynamical games. With the help of computer modeling conditions favoring to increase of the market share of the new united carrier are determined. 